\title{
ASSURANCE DOS RELATÓRIOS DE SUSTENTABILIDADE NO BRASIL: QUAIS FATORES PODEM INFLUENCIAR A SUA OCORRÊNCIA?
}

\section{SUSTAINABILITY REPORTING ASSURANCE IN BRAZIL: WHAT FACTORS INFLUENCE OCCURRENCE?}

\section{FACTORES QUE INFLUYEN EN LA GARANTÍA DE LOS INFORMES DE SOSTENIBILIDAD EN BRASIL: QUÉ FACTORES PUEDEN INFLUIR EN SU APARICIÓN?}

Recebido em: 27/03/2019

Avaliado em: 26/08/2019

Reformulado em: 13/09/2019

Aceito para publicação em: 13/09/2019

Publicado em: 02/09/2020

Editor Responsável: Moacir M. Rodrigues Junior
Gabriela Borges Silveira ${ }^{1}$

Luiz Alberton ${ }^{2}$

\section{RESUMO}

A asseguração externa tem sido empregada no sentido de atribuir credibilidade aos relatórios de sustentabilidade (RS), de modo a influenciar a percepção sobre o nível de legitimidade das organizações. Desse modo, esta pesquisa teve como objetivo identificar os fatores que podem influenciar a ocorrência da asseguração dos RS. A amostra deste estudo compreende as empresas brasileiras listadas na B3 em 2017 e que divulgaram seus RS durante o período de 2012 a 2016 . Por meio da técnica de regressão logística em painel de efeitos mistos, buscou-se testar a relação entre a probabilidade da adoção da asseguração dos RS e as variáveis Setor Ambientalmente Sensível, Pressão do Setor, Comitê de Sustentabilidade, Global Reporting Initiative e Oportunidade de Crescimento, além das variáveis de controle apontadas pela literatura, que são: Tamanho, Rentabilidade, Endividamento e Setor. De acordo com os resultados, Pressão do Setor, Comitê de Sustentabilidade, Diretrizes da GRI e Tamanho são fatores que podem influenciar a adoção da asseguração dos RS. Esses achados sugerem que a busca das empresas por legitimidade por meio dessa prática ocorre em empresas maiores e mais evidentes, e pode resultar de práticas amplamente adotadas em seu setor, do monitoramento das questões de sustentabilidade exercido por comitês especializados ligados à estrutura de governança corporativa e da adoção de práticas de relato que atribuem qualidade e credibilidade aos RS.

Palavras-chave: Relatório de Sustentabilidade. Asseguração Externa. Legitimidade.

\section{ABSTRACT}

External assurance has been employed to give sustainability reporting SR credibility and influence the perception of the level of legitimacy of organizations. Thus, this study aimed to identify the factors that influence the occurrence of SR assurance. The sample of this study comprises the Brazilian

\footnotetext{
1 Doutoranda e Mestre em Contabilidade pela Universidade Federal de Santa Catarina. E-mail: gabi_confiante@yahoo.com.br

${ }^{2}$ Doutor em Engenharia de Produção pela Universidade Federal da Universidade Federal de Santa Catarina. Professor do Departamento de Contabilidade e do Programa de Pós-Graduação em Contabilidade da Universidade Federal de Santa Catarina. E-mail: luiz.alberton@ufsc.br
} 
companies listed on B3 in 2017 and that disclosed RS, from 2012 to 2016 . The mixed effects panel data technique was applied to test the relationship between the likelihood of adopting SR assurance and the Environmentally Sensitive Sector, Sector Pressure, Sustainability Committee, Global Reporting Initiative and Growth Opportunity variables. In addition to the control variables mentioned in the literature Size, Profitability, Liability and Sector. The results revealed that the Sector Pressure, Sustainability Committee, Global Reporting Initiative and Size are factors that influence the likelihood of adopting RS assurance. These findings suggest that companie's search for legitimacy through this practice occurs in larger and more evident companies and may result from practices widely adopted in their sector, from the monitoring of sustainability issues exercised by specialized committees linked to the corporate governance structure and the adoption of reporting practices that attribute quality and credibility to SR.

Keywords: Sustainability Report. External Assurance. Legitimacy.

\section{RESUMEN}

La garantía externa se ha empleado para dar credibilidad a los informes de sostenibilidad (SI) con el fin de influir en la percepción del nivel de legitimidad de las organizaciones. Por lo tanto, esta investigación busca identificar los factores que pueden influir en la ocurrencia de la garantía de SI. La muestra de este estudio comprende las empresas brasileñas que figuran en B3 en 2017 y que divulgaron SI durante el período de 2012 a 2016. A través de la técnica de regresión logística del panel de efectos mixtos, buscamos probar la relación entre la probabilidad de adoptar la garantía de SI y las variables Sector ambientalmente sensible, Presión del sector, Comité de sostenibilidad, Global Reporting Initiative y Oportunidad de crecimiento. Además de las variables de control mencionadas en la literatura Tamaño, Rentabilidad, Endeudamiento y Sector. Según los resultados, la presión del sector, el comité de sostenibilidad, las pautas GRI y el tamaño son factores que pueden influir en la probabilidad de adoptar la garantía de SI. Estos hallazgos sugieren que la búsqueda de legitimidad de las compañías a través de esta práctica ocurre en compañías más grandes y más evidentes y puede ser el resultado de prácticas ampliamente adoptadas en su industria, el monitoreo de problemas de sostenibilidad por parte de comités especializados vinculados a la estructura de gobierno corporativo y adopción de prácticas de informes que atribuyen calidad y credibilidad a SI.

Palabras-clave: Informe de Sostenibilidad. Garantía Externa. Legitimidad.

\section{INTRODUÇÃO}

Ao considerar a demanda informacional crescente de diversos stakeholders sobre os impactos ambientais e sociais das atividades empresariais, os Relatórios de Sustentabilidade (RS), como parte ou mecanismo da Responsabilidade Social Corporativa, têm se estabelecido firmemente como componentes da divulgação corporativa, como relatado pela pesquisa da KPMG (2011) em que 95\% das 250 maiores corporações do mundo publicam separadamente. Dessa maneira, atualmente, os RS apresentam-se como relatórios autônomos, chamados de stand-alone, configurando-se em um importante instrumento de comunicação entre as organizações e suas partes interessadas (Cho, Michelon, Patten, \& Roberts, 2014).

Desse modo, com a evolução da divulgação dos RS, surge o questionamento sobre a credibilidade e qualidade desses relatórios (Martínez-Ferrero \& García-Sánchez, 2016; Cho et al., 2014; Kolk \& Perego, 2010; Simnett, Vanstraelen \& Chua, 2009). Gürtürk e Hahn (2016) explicam que, nas questões relacionadas à sustentabilidade, a empresa cria uma forte dependência da percepção e pressão de seus stakeholders externos ao atuar em um cenário no qual a organização reage a essas expectativas, fornecendo informações e a garantia destas. Isso favorece o argumento de que a verificação externa - opinião de um profissional independente - não somente reforça a qualidade mas também melhora a credibilidade dos RS (Kuzey \& Uyar, 2017; Moroney, Windsor \& Aw, 2012). 
No âmbito geral, a asseguração dos RS é considerada uma prática voluntária por parte das empresas. Silveira, Ferreira, Farias, Van Bellen, e Rover (2019) e Simnett et al. (2009) constataram que, embora possam existir certos requisitos para a divulgação das informações não financeiras para determinados grupos - tais como aqueles regulados por agentes específicos -, não há regulamento que requeira a publicação dos RS de forma separada das demais demonstrações. E, da mesma forma, não existe regulamento que exija que as informações neste relatório sejam asseguradas.

Segundo "Focal Point Brazil" (GRI, 2017), o Brasil ocupa o terceiro lugar no mundo em número de empresas que divulgam os RS; em 2010, mais 160 relatórios brasileiros nos moldes da GRI foram registrados na lista de relatórios da GRI. Porém, Hodge, Subramaniam e Stewart (2009) questionaram esse aumento do número de divulgação dos RS sem o devido aumento do nível de confiança pública. Deve-se mencionar, ainda, que problemas relacionados à falta de uniformidade e regularidade da divulgação dos RS podem afetar a confiabilidade da informação. Neste ponto, Martínez-Ferrero e García-Sánchez (2016) ressaltam que, dentro de um contexto em que a informação de sustentabilidade é certamente questionável ou dificilmente credível, empresas podem "voluntariamente" iniciar um processo de verificação dessas informações. Diante disso, Simnett et al. (2009) defendem a asseguração dos RS como critério de confiabilidade.

Kim, Park e Wier (2012) e Hemingway e Maclagan (2004) alertam que os gestores podem envolver-se em práticas de responsabilidade social para cobrir o impacto da má conduta corporativa, ou seja, o desenvolvimento de atividades relacionadas à Responsabilidade Social Corporativa atuaria como uma forma de "seguro" da reputação, dando a impressão às partes interessadas de que a firma é transparente, quando, na verdade, se trataria de pura aparência.

Acerca da ótica da Teoria da Legitimidade, entende-se que a legitimidade social consiste na principal razão pela qual uma organização deve divulgar os RS (Eugenio, 2010; Arvidsson, 2010). E, à medida que imposições institucionais e pressões políticas e sociais demandam mudanças no nível de legitimidade das organizações (Sancovschi \& Silva, 2006), a asseguração dos RS tem sido empregada no sentido de atribuir credibilidade a este tipo de relatório, o que, possivelmente, poderia aumentar o nível de legitimidade das organizações (O’Dwyer, Owen \& Unerman, 2011).

Ao considerar que a asseguração externa tem sido empregada no sentido de atribuir credibilidade aos RS, de modo a influenciar a percepção sobre o nível de legitimidade das organizações, busca-se responder à seguinte questão de pesquisa: quais fatores podem influenciar a ocorrência da asseguração dos relatórios de sustentabilidade no Brasil? Assim, o estudo objetiva identificar os fatores que podem influenciar a ocorrência da asseguração dos relatórios de sustentabilidade das empresas brasileiras listadas na B3 em 2017 no período de 2012 a 2016.

Simnett et al. (2009) argumentam que empresas com uma maior necessidade de aumentar a credibilidade dos seus RS e construir sua boa reputação corporativa são mais propensas a ter seus relatórios assegurados. E esta necessidade é decorrente de fatores relacionados às características das empresas, do setor de atuação e do país. Dentre essas possibilidades, o estudo buscou evidenciar a influência de fatores em nível de empresa e setor que caracterizam a necessidade do reforço no nível de legitimidade das organizações. Por exemplo, as empresas que pertencem a setores de maiores impactos ambientais e sociais, o que, também, exige um maior nível de divulgação socioambiental (Adams, Hill \& Roberts, 1998; Patten, 2002), e, ainda, empresas sujeitas a pressões resultantes de forças miméticas, por exemplo, a pressão do setor, possuem maior necessidade de reforçar a legitimidade de suas operações por meio da asseguração externa (Martínez-Ferrero \& GarcíaSánchez, 2016; Peters \& Romi, 2014; Simnett et al., 2009).

O estudo justifica-se pela necessidade de reforçar os pilares teórico-empíricos a respeito do papel da asseguração externa dos RS e a legitimidade das organizações, pressupondo que a principal motivação para assegurar os RS parece envolver fatores relacionados à legitimidade de suas operações, e, consequentemente, à construção de sua reputação corporativa perante a sociedade e os demais stakeholders. Assim, com esta investigação, busca-se analisar estes fatores em um contexto diverso dos estudos já realizados em âmbito internacional (Martínez-Ferrero \& García-Sánchez, 
2016, 2017; Branco, Delgado, Gomes, \& Eugénio, 2014; Cho et ; al., 2014; Ruhnke \& Gabriel, 2013; Perego \& Kolk, 2012; Gomes, 2012; Kolk \& Perego, 2010; Simnett et al., 2009). Também pode contribuir com os poucos estudos em países emergentes, sobretudo em empresas brasileiras (Silveira et al., 2019; Silveira, Alberton \& Vicente, 2017; Kuzey \& Uyar, 2017; Luna, Gomes, Martinez, Cordeiro Filho, Bomfim, 2017).

Justifica-se, ainda, o estudo pelo incremento nas discussões sobre o papel da Contabilidade Socioambiental e sua natureza interdisciplinar, a qual requer a ligação e a cooperação entre as disciplinas: contábeis, sociais e ecológicas. Para isso, é fundamental construir um diálogo aberto para facilitar os discursos interdisciplinares e, posteriormente, elaborar e auditar informações sobre o desempenho socioambiental das organizações (Lamberton, 2005).

\section{REFERENCIAL TEÓRICO}

\subsection{Teoria da Legitimidade e Asseguração dos Relatórios de Sustentabilidade}

Suchman (1995) conceitua legitimidade como uma percepção ou suposição generalizada de que as ações de uma entidade são desejáveis e apropriadas dentro de um sistema socialmente constituído de normas, valores e crenças. Deegan (2002) explica que o contrato social é a premissa central da legitimidade, uma vez que as organizações possuem contratos implícitos com a sociedade; logo, cumprir este contrato legitima a própria entidade, bem como suas operações. E isto implica conhecer e aderir às expectativas de um sistema social (Deephouse \& Carter, 2005).

A busca da legitimidade pelas organizações é incentivada pela continuidade e credibilidade de suas operações. Assim, a legitimidade melhora a estabilidade e a compreensão das atividades organizacionais, o que leva à persistência e afeta a percepção do público sobre a empresa, uma vez que o público é mais susceptível de fornecer recursos para as organizações que apresentam comportamento tido como apropriado ou adequado (Parsons, 1960; Suchman, 1995).

Nessa perspectiva, a Teoria da legitimidade tem sido amplamente utilizada pela literatura para explicar a divulgação de informações de natureza voluntária, como aquelas relativas ao desempenho ambiental e social ou de sustentabilidade. Eugenio (2010) afirma que tornar conhecida a maneira como a empresa se relaciona com os aspectos ambientais e sociais pode ser vista como uma forma de legitimar as atividades e preservar a continuidade da organização. Meyer e Rowan (1977) explicam que, para atingir seus objetivos de continuidade no mercado, as empresas podem adotar diversas práticas e procedimentos para legitimar suas atividades. Isso em razão de que as empresas se empenham em assegurar que suas ações sejam percebidas pelos diversos stakeholders como legítimas (Islam \& Deegan, 2008).

Segundo Sancovschi e Silva (2006), as imposições institucionais ou regulatórias influenciam no surgimento de mudanças no nível de legitimidade das organizações. Certas ações podem aumentar ou diminuir essa legitimidade, resultando em consequências materiais para uma empresa (Suchman, 1995). Diante disso, a asseguração dos RS tem sido empregada no sentido de atribuir credibilidade a este tipo de relatório, o que, possivelmente poderia aumentar o nível de legitimidade das organizações (O’Dwyer et al., 2011). Dessa forma, as empresas procuram assegurar que o conteúdo divulgado em seus relatórios é verdadeiro, o que foi objeto de revisão por terceiros (Hummel, Schlick \& Fifka, 2019).

Simnett et al. (2009) evidenciaram que as empresas que operam em indústrias que enfrentam maior risco social e ambiental mostram uma maior adesão da asseguração das informações de sustentabilidade, usando a sua declaração de garantia como uma ferramenta para melhorar a sua reputação e credibilidade.

Peters e Romi (2014) afirmam que empresas de setores ambientalmente sensíveis (SAS) suportam maiores pressões políticas e sociais relacionadas às questões de sustentabilidade, como também criam uma maior apreensão externa sobre a credibilidade de suas informações, e, assim, existe uma maior necessidade de fornecer uma garantia. Já Cho et al. (2014) investigaram a relação 
entre garantia dos RS e Tamanho; Setor Ambientalmente Sensível; Setor Financeiro; Extensão dos Relatórios; e Valor de Mercado. Porém, os resultados encontrados mostraram que somente Extensão dos Relatórios e Setor Ambientalmente Sensível influenciam a asseguração dos RS.

Com base no que foi exposto, supõe-se que as empresas pertencentes aos SAS no mercado brasileiro necessitam aumentar a credibilidade dos RS, com o intuito de legitimar suas operações por meio da asseguração externa. Desse modo, formula-se a seguinte hipótese:

H1: A probabilidade de adoção da asseguração dos RS é afetada positivamente pelo impacto ambiental causado pelo setor de atividade.

Além da necessidade de aumentar a confiança do usuário, as empresas podem estar sujeitas a um ambiente de legitimação, porque a legitimidade das instituições pode resultar de comportamentos isomórficos, visto que uma prática é amplamente adotada em uma mesma indústria, em mesmo setor ou país (Meyer \& Rowan, 1977).

Convém salientar que Martínez-Ferrero e García-Sánchez (2016), com base na teoria neoinstitucional, analisaram se a asseguração dos relatórios de sustentabilidade deriva de forças coercivas, normativas e miméticas relacionadas com a força jurídica, cultural e setorial, respectivamente. Ao verificarem uma amostra com 696 empresas de diversos países, em análise de dados em painel para o período de 2007-2014, esses autores verificaram que a garantia voluntária atua como ferramenta de legitimação implementada pelas empresas nos países que têm maior força coercitiva, desenvolvimento cultural, setores e desenvolvem melhor suas práticas de sustentabilidade, ou seja, divulgam e asseguram seus RS.

Ademais, Peters e Romi (2014) constataram que as empresas apresentam comportamento de isomorfismo mimético, ao se tornarem semelhantes em suas práticas de asseguração quando confrontadas com maior Pressão do Setor. Portanto, tendo como proposição de que as empresas do mercado brasileiro estão condicionadas à pressão exercida, relativa à asseguração dos RS, de seus setores de atuação tem-se a seguinte hipótese de pesquisa:

H2: A probabilidade de adoção da asseguração dos RS é afetada positivamente pela Pressão do Setor.

No que se refere ao nível de legitimidade das organizações, Ruhnke e Gabriel (2013) argumentam que empresas com mais experiência e qualidade na divulgação dos RS são mais propensas a procurar a asseguração externa. E, ao verificarem os determinantes da garantia externa das empresas da Alemanha, Holanda e Grã-Bretanha, esses autores encontraram significância para os Níveis de Aplicação do GRI, Departamento de Sustentabilidade e Tamanho. Dessa maneira, a existência de comitê sustentabilidade pode ser vista como uma proxy para uma melhor integração das questões de sustentabilidade no planejamento estratégico e operacional da gestão empresarial, enfatizando a importância da divulgação dos RS e, consequentemente, da credibilidade das informações reportadas.

Martínez-Ferrero e García-Sánchez (2017) também examinaram o efeito dos mecanismos de governança corporativa na decisão das empresas em assegurar seus RS em países com maior orientação para os stakeholders para uma amostra internacional de 610 empresas do período 20072014. Os resultados do estudo fornecem evidências de que a garantia dos RS estão positivamente associadas à Independência do Conselho e à Atividade do Comitê de Sustentabilidade.

Nesse sentido, segundo Peters e Romi (2014), as empresas veem desenvolvendo práticas de governança corporativa com o objetivo de gerenciar e monitorar as questões de sustentabilidade, instituindo comitês específicos no Conselho da Administração. Os autores explicam que, devido à função de monitoramento informacional desses comitês, a asseguração externa poderia ser vista como um meio para potencializar a legitimidade das atividades do comitê perante a alta administração e os demais stakeholders. 
Assim, esta pesquisa investiga a premissa inicial de que, no mercado brasileiro, as empresas que dispõem de um comitê que trata dos assuntos relativos à sustentabilidade tendem a assegurar os seus RS, dada a seguinte hipótese:

H3: A probabilidade de adoção da asseguração dos RS é afetada positivamente pela existência do Comitê de Sustentabilidade.

É importante perceber que as características de elaboração do relatório podem influenciar a probabilidade de adoção da garantia voluntária (Ruhnke \& Gabriel, 2013), uma vez que a asseguração externa dos RS também é recomendada pelos repórteres de sustentabilidade - por exemplo, a Global Reporting Initiative (GRI) - que ressaltam o atributo de credibilidade aos RS, proporcionado pelos serviços de garantia. A GRI constitui-se em um conjunto de diretrizes para RS, de natureza não governamental, que abrange as dimensões econômica, social e ambiental, sendo consideradas as ferramentas de comunicação mais legítimas e difundidas sobre as práticas socioambientais no mercado, visando ao desenvolvimento econômico sustentável (Gurvitsh \& Sidorova, 2012; Lozano \& Huisingh, 2011). Logo, para inferir sobre a qualidade dos RS, é possível utilizar a adesão de uma empresa às diretrizes da GRI.

Quanto a esse aspecto, Lock e Seele (2016) descobriram que a padronização por meio da estrutura GRI melhora a qualidade dos relatórios pelo fato de serem encontradas evidências de que um nível elevado de aplicação, quanto à emissão de relatórios com base na GRI, afeta a credibilidade dos relatórios positivamente. Além disso, Runke e Gabriel (2013) asseveram que, na perspectiva do prestador de garantia, torna-se vantajoso quando o RS é elaborado de acordo com diretrizes padronizadas de sustentabilidade; afinal, os possíveis desvios e as distorções podem ser mais facilmente identificados a partir de determinados critérios derivados dos padrões de divulgação dos relatórios.

Silveira et al. (2019) identificaram os fatores associados à asseguração dos RS das empresas brasileiras listadas no índice IbrX-100 da B3 no período de 2011-2015. Os resultados permitiram aos autores concluírem que empresas de setores regulados, pertencentes ao ISE e que seguem as diretrizes propostas pelo GRI tendem a assegurar os RS, e que, ainda, tomam por relevante o trabalho ser prestado por empresas de auditoria. Os autores enfatizam, ainda, a baixa adoção da verificação externa, ao verificarem que apenas $49 \%$ da amostra asseguraram seus relatórios.

Dessa forma, supondo que as empresas que atribuem qualidade aos RS ao aderirem às diretrizes GRI recebem o incentivo de que podem reforçar a credibilidade de seus relatórios por meio da asseguração externa, propõe-se a seguinte hipótese:

H4: A probabilidade de adoção da asseguração dos RS é afetada positivamente pela adesão das diretrizes da GRI.

As empresas em constante crescimento, no sentido de demostrar seus objetivos de continuidade no mercado, são mais susceptíveis de incoporar práticas comerciais sustentáveis em suas estratégias de negócios (Artiach, Lee, Nelson, \& Walker, 2010). Os RS poderiam desempenhar um papel de legitimação nas operações de empresas em crescimento; desse modo, a sustentabilidade econômica recebe o suporte dos aspectos ambiental e social como condição de preocupação para um crescimento sustentável (Kuzey \& Uyar, 2017).

Kuzey e Uyar (2017) investigaram o impacto dos fatores delimitados em Tamanho, Indústria, Alavancagem Financeira, Rentabilidade, Liquidez, Fluxo de Caixa Livre, Oportunidade de Crescimento e Free-Float na adoção da asseguração dos RS das companhias de capital aberto da Turquia, e levantaram a hipotese de que empresas em crescimento são mais propensas a publicar os RS em conformidade com as diretrizes da GRI, bem como submetê-los à asseguração externa, a fim 
de legitimar suas atividades. No entanto, os resultados do estudo de Kuzey e Uyar (2017) não apontaram significância para variável Oportunidade de Crescimento.

Com objetivo de testar essa premissa em contexto brasileiro, formula-se a seguinte hipótese:

H5: A probabilidade de adoção da asseguração dos RS é afetada positivamente pela Oportunidade de Crescimento das empresas.

Portanto, percebe-se a prática de asseguração dos RS como parte do processo de legitimação das organizações perante seus stakeholders, o que motivou essa pesquisa a investigar as características relacionadas a esse ambiente de legitimação vivenciado pelas empresas brasileiras.

\section{PROCEDIMENTOS METODOLÓGICOS}

\subsection{População, Amostra e Dados Coletados}

A população deste estudo é constituída por companhias brasileiras de capital aberto listadas na Brasil, Balcão e Bolsa (B3) em 2017. A amostra, por sua vez, é composta por empresas que publicaram os RS em formato stand-alone relativos ao período de 2012 a 2016 . O motivo para a seleção desse espaço de tempo se justifica pelo "Comunicado Externo 017/2011-DP” da B3, o qual orienta que as empresas listadas relatem, a partir de 2012, em seu Formulário de Referência (item 7.8 - Políticas socioambientais), se realizam a divulgação de RS ou Relato Integrado; e, em caso negativo, elaborem sua justificativa.

Após a identificação das empresas, optou-se por excluir da amostra aquelas que não divulgam os relatórios de forma anual, em razão de que, em geral, esse tipo de relatório é divulgado de forma voluntária e não segue padrões regulares de divulgação, havendo empresas que divulgam seus relatórios de forma bianual. Da mesma forma, há companhias que publicam os RS de forma consolidada, ou seja, com divulgação de um único relatório para um determinado grupo econômico; assim, o critério utilizado é de considerar apenas os dados da empresa que efetivamente divulgou o relatório, isto é, da companhia controladora. A Tabela 1 apresenta o processo de seleção da amostra para o período de análise.

Tabela 1 - Seleção da amostra (2012 a 2016)

\begin{tabular}{lccccc}
\hline \multicolumn{1}{c}{ Descrição } & $\mathbf{2 0 1 2}$ & $\mathbf{2 0 1 3}$ & $\mathbf{2 0 1 4}$ & $\mathbf{2 0 1 5}$ & $\mathbf{2 0 1 6}$ \\
\hline Empresas brasileiras listadas na B3 em 2017 & 446 & 446 & 446 & 446 & 446 \\
\hline (-) Empresas que não divulgaram RS & 309 & 300 & 304 & 302 & 297 \\
\hline (-) Empresas que não atenderam aos critérios da pesquisa* & 40 & 44 & 43 & 41 & 55 \\
\hline Total da Amostra & $\mathbf{9 7}$ & $\mathbf{1 0 2}$ & $\mathbf{9 9}$ & $\mathbf{1 0 3}$ & $\mathbf{9 4}$ \\
\hline
\end{tabular}

Nota.* Critérios da pesquisa: relatórios de sustentabilidade em formato stand-alone disponíveis nos websites das empresas e de periocidade de publicação anual.

Fonte: Elaborada pelos autores (2019).

Como mostra a Tabela 1, nota-se que a composição da amostra não foi a mesma para todos os anos e a quantidade de companhias participantes da pesquisa foi de 119. Em suma, este estudo partiu das 446 empresas listadas em bolsa em 2017 e verificou, para cada ano da análise (2012-2016), se estas publicaram ou não os RS. Ressalta-se que, referente ao ano de 2016, a amostra foi composta pelas empresas que divulgaram seus relatórios até a data de 25 de setembro de 2017 , considerando a divulgação e periocidade não obrigatória dos RS. 


\subsection{Definição das Variáveis}

\subsubsection{Variável dependente}

A variável dependente ASSEG assumida consiste em uma variável binária ou nominal codificada como 0 e 1 (dummy). Dessa forma, quando a empresa assegura o seu RS é atribuído 1 e para os casos em que as empresas não asseguram os RS atribui-se 0. Ressalta-se que os RS foram considerados assegurados quando a empresa disponibilizou a carta de asseguração externa emitida formalmente pela prestadora de garantia em anexo ao seu RS.

\subsubsection{Variável independente}

As variáveis utilizadas para explicar a decisão das empresas em assegurar seus RS sob a ótica da Teoria da Legitimidade foram definidas de acordo com a literatura e as características das empresas da amostra. No Quadro 1, apresentam-se as definições dessas métricas.

Quadro 1 - Definições das varáveis independentes da pesquisa

\begin{tabular}{|c|c|c|c|c|}
\hline Váriável & Proxy & Referências & Hipótese & Fonte \\
\hline $\begin{array}{l}\text { Setor } \\
\text { Ambientalmente } \\
\text { Sensível (SAS) }\end{array}$ & $\begin{array}{l}\text { Números Naturais de } 1 \text { a } 4 \text {, } \\
\text { representando setores de atividade das } \\
\text { empresas que possuem classificação de } \\
\text { acordo com a Lei no } 10.165 / 2000 \\
\text { (Brasil, 2000), os quais são: Não se } \\
\text { aplica (1), Baixo Impacto (2), Médio } \\
\text { Impacto (3) e Alto Impacto (4). }\end{array}$ & $\begin{array}{l}\text { Cho et al. }(2014) \text {; } \\
\text { Peters e Romi } \\
\text { (2014); Simnett et } \\
\text { al. (2009). }\end{array}$ & $\mathrm{H} 1$ & $\begin{array}{l}\text { Lei } n^{\circ} 10.165 / 2000 \\
\text { (BRASIL, 2000); } \\
\text { Sítio eletrônico da } \\
\text { B3. }\end{array}$ \\
\hline $\begin{array}{l}\text { Pressão do Setor } \\
\quad \text { (PRESET) }\end{array}$ & $\begin{array}{l}\text { Percentual }(\%) \text { de empresas que } \\
\text { pertencem ao mesmo setor que } \\
\text { asseguram o RS. }\end{array}$ & $\begin{array}{l}\text { Martínez-Ferrero } \\
\text { e García-Sánchez } \\
\text { (2016); Peters e } \\
\text { Romi (2014). }\end{array}$ & $\mathrm{H} 2$ & $\begin{array}{l}\text { Sítio eletrônico da } \\
\text { B3 e RS. }\end{array}$ \\
\hline $\begin{array}{l}\text { Comitê de } \\
\text { Sustentabilidade } \\
\text { (COMITE) }\end{array}$ & $\begin{array}{l}\text { Variável dummy, sendo } 1 \text { para empresas } \\
\text { que possuem Comitê de } \\
\text { Sustentabilidade e } 0 \text { caso contrário. }\end{array}$ & $\begin{array}{l}\text { Peters e Romi } \\
\text { (2014); Ruhnke e } \\
\text { Gabriel (2013). }\end{array}$ & H3 & $\begin{array}{l}\text { No RS, no item } \\
\text { destinado à } \\
\text { Governança } \\
\text { Corporativa. } \\
\end{array}$ \\
\hline $\begin{array}{l}\text { Global Reporting } \\
\text { Initiative (GRI) }\end{array}$ & $\begin{array}{l}\text { Variável dummy, sendo } 1 \text { para as } \\
\text { empresas que utilizam as Diretrizes da } \\
\text { GRI para a elaboração dos RS e } 0 \text { para } \\
\text { aquelas que não as utilizam. }\end{array}$ & $\begin{array}{l}\text { Silveira et al. } \\
\text { (2019); Ruhnke e } \\
\text { Gabriel (2013). }\end{array}$ & $\mathrm{H} 4$ & $\mathrm{RS}$ \\
\hline $\begin{array}{l}\text { Oportunidade de } \\
\text { Crescimento } \\
\text { (CRESC) }\end{array}$ & $\begin{array}{l}\text { Variação da Receita Operacional ou } \\
\text { Financeira Líquida entre t e } \mathrm{t}-1 \text {. }\end{array}$ & $\begin{array}{l}\text { Kuzey e Uyar } \\
\text { (2017); Artiach et } \\
\text { al. (2010). }\end{array}$ & H5 & Economática ${ }^{\circledR}$ \\
\hline
\end{tabular}

Fonte: Elaborado pelos autores (2019).

Destaca-se que a literatura (Simnett et al., 2009; Peters \& Romi, 2014; Cho et al., 2014) definiu como SAS as empresas pertencentes aos setores de Óleo (exploração de petróleo), papel, químico (produtos químicos e aliados) petróleo (refinação de petróleo), metais e utilidades (serviços públicos). No entanto, com intuito de avaliar o cenário brasileiro e testar a Hipótese 1, este estudo optou por classificar as empresas conforme a Lei $n^{\circ}$ 10.165/2000 (BRASIL, 2000) que dispõe sobre a Política Nacional do Meio Ambiente.

Para PRESET, semelhante aos estudos de Peters e Romi (2014) e Martínez-Ferrero e GarcíaSánchez (2016), quando confrontadas com maior Pressão do Setor, as empresas tendem a assegurar os RS. Desse modo, utiliza-se como proxy de Pressão do Setor (PRESET) o percentual (\%) de empresas que pertencem ao mesmo setor que asseguram os RS, dada pela razão entre a quantidade de empresas que asseguram os RS e a população do setor.

Em relação à variável CRESC, Kuzey e Uyar (2017), ao investigarem a adoção da asseguração dos RS das empresas turcas, utilizaram o índice Market-to-book; porém, não puderam constatar a 
significância desta variável no modelo proposto. Assim, de forma alternativa, este estudo optou por proxy a variação da Receita Operacional Líquida entre t e $\mathrm{t}$ - 1 . Ressalta-se que, para as instituições bancárias, fez-se o uso da variação da Receita de Intermediação Financeira.

\subsubsection{Variável de controle}

As empresas maiores e mais rentáveis tendem a ter recursos financeiros necessários para promover atividades ligadas ao desenvolvimento sustentável, bem como emitir e assegurar os respectivos relatórios (Branco et al., 2014). Neste sentido, devido à restrição dos custos que a asseguração dos RS impõe às empresas, pondera-se que as que possuem maior grau de endividamento tendem a não assegurar o relatório.

Ressalta-se que fatores específicos de cada país também podem influenciar a demanda pela garantia voluntária (Ruhnke \& Gabriel, 2013). No caso das companhias brasileiras, observou-se que os setores de Energia Elétrica e Bancos seguem orientações de suas agências reguladoras quanto à divulgação de informações socioambientais - Despacho no 3.034/2006 da Agência Nacional de Energia Elétrica (ANEEL) e a Resolução CMN nº 4.327/2014 do Banco Central. E é possível que estas empresas estejam sob uma maior pressão dos grupos das partes interessadas nestes setores e, desse modo, necessitem oferecer credibilidade para as informações reportadas, reforçando o seu nível de legitimidade (Cho et al., 2014; Kolk \& Perego, 2010).

Seguindo estudos anteriores que utilizaram essas variáveis, como Simnett et al., (2009), Ruhnke e Gabriel (2013), Cho et al. (2014), Branco et al. (2014), Peters e Romi (2014), MartínezFerrero e García-Sánchez (2016) e Martínez-Ferrero e García-Sánchez (2017), as variáveis de controle são operacionalizadas da seguinte maneira: a) Tamanho (TAM) - logaritmo do ativo total; b) Rentabilidade (RENT) - Retorno sobre os Ativos (ROA); c) Endividamento (ENDIV) - a soma do Passivo Circulante e Não Circulante divido pelo Ativo Total; e d) Setor (SET) - corresponde a uma variável dummy (0 e 1) que diferencie as empresas do setor de Energia Elétrica e Bancos.

As variáveis TAM, RENT e ENDIV foram coletadas no banco de dados do Economática®. Enquanto a variável SET foi classificada conforme o sítio eletrônico da B3.

\subsection{Técnica de Análise dos dados}

A técnica estatística utilizada consistiu em um modelo de regressão logística em painel para investigar o efeito das variáveis pelas quais os indivíduos, objetos ou sujeitos estão expostos sobre a probabilidade de ocorrência de determinado evento de interesse que se modifica entre indivíduos durante um espaço de tempo, o qual, nesta pesquisa, se atribui à asseguração dos RS. Na regressão logística, a variável dependente é nominal e assume valor de 1 ou $0 \mathrm{com}$ base na existência ou ausência de uma determinada característica qualitativa, sendo, portanto, a técnica indicada para analisar a decisão das empresas em assegurar 1 ou não 0 os RS (Fávero, Belfiore, Silva, \& Chan, 2009).

Ressalta-se que os modelos logísticos possuem as seguintes premissas: relação linear entre o vetor das variáveis explicativas e a variável dependente; valor esperado dos resíduos é igual a 0 ; ausência de heterocedasticidade; e ausência de multicolinearidade. Porém, não pressupõem a existência de homogeneidade de variância e normalidade dos resíduos, pois se fundamentam no método de máxima verossimilhança, isto é, buscam estimar a probabilidade de ocorrência de um evento (Fávero et al., 2009). Dessa forma, esses modelos dispensam os testes dos pressupostos para a normalidade empregados normalmente em modelos lineares.

Utilizou-se como abordagem para dados em painel a regressão multinível na forma de modelo de efeitos mistos. Segundo Hox (2010), esse modelo busca estimar com eficiência o erro padrão dos coeficientes de regressão, pois leva em consideração a suposição de que as observações dentro de um conglomerado possam estar correlacionadas. Além de analisar a influência dos efeitos contextuais, 
fixos ou aleatórios, observando as variáveis independentes da empresa i que variam no tempo (nível 1) e variáveis independentes da empresa i fixas no tempo (nível 2).

O modelo dos fatores que podem influenciar a probabilidade da adoção da asseguração dos RS das companhias brasileiras está descrito na equação 1:

$$
\begin{aligned}
& \log \left[\frac{\operatorname{prob}\left(A S S E G_{i t}\right)}{1-\operatorname{prob}\left(A S S E G_{i t}\right)}\right]=\alpha_{i}+\beta_{1} S A S_{i t}+\beta_{2} P_{R E S E T} i t+\beta_{3} \operatorname{COMITE}_{i t}+\beta_{4} G R I_{i t}+ \\
& \beta_{5} \text { CRESC }_{i t}+\beta_{6} \text { TAM }_{i t}+\beta_{7} R E N T_{i t}+\beta_{8} E N D I V_{i t}+\beta_{9} S E T_{i t}+\varepsilon_{i t} \text { (1) }
\end{aligned}
$$

Em que $A_{S S E G}$ it representa a variável dependente binária que representa a asseguração (1) e não asseguração dos RS; $\alpha_{i}$ o intercepto da equação; $\beta_{1}, \beta_{2}, \beta_{3}, \beta_{4}, \beta_{5}, \beta_{6}, \beta_{7}, \beta_{8}$ e $\beta_{9}$ os receptivos parâmetros estimados com as variáveis independentes Setor Ambientalmente Sensível, Pressão do Setor, Comitê de Sustentabilidade, GRI, Oportunidade de Crescimento, Tamanho, Rentabilidade, Endividamento e Setor; e $\varepsilon_{i t}$ o termo aleatório da regressão.

$\mathrm{O}$ tratamento dos dados incluiu a utilização de planilhas eletrônicas e o uso dos softwares estatísticos $R$ versão 3.6.0 for Windows, pacotes "lme4" e "nlme", e Stata® 15.

\section{DESCRIÇÃO E ANÁLISE DOS RESULTADOS}

\subsection{Análise descritiva das variáveis}

Para as variáveis binárias utilizadas para testar H1, H3 e H4, apresenta-se a Tabela 2 com as frequências e os percentuais das variáveis, considerando, ao longo do tempo, a variação entre indivíduos (between) e a variação para cada indivíduo (within).

\begin{tabular}{|c|c|c|c|c|c|c|}
\hline \multirow{2}{*}{\multicolumn{2}{|c|}{ Variáveis }} & \multicolumn{2}{|c|}{ Geral } & \multicolumn{2}{|c|}{ Between } & \multirow{2}{*}{$\begin{array}{c}\text { Within } \\
\text { Proporção }\end{array}$} \\
\hline & & Frequência & Proporção & Frequência & Proporção & \\
\hline \multirow{3}{*}{ ASSEG } & 0 & 296 & 59,80 & 84 & 70,59 & 89,94 \\
\hline & 1 & 199 & 40,20 & 50 & 42,02 & 86,90 \\
\hline & Total & 495 & 100,00 & 134 & 112,61 & 88,81 \\
\hline \multirow{3}{*}{ SAS 1} & 0 & 225 & 45,45 & 55 & 46,20 & 100,00 \\
\hline & 1 & 270 & 54.55 & 64 & 53,80 & 100,00 \\
\hline & Total & 495 & 100,00 & 119 & 100,00 & 100,00 \\
\hline \multirow{3}{*}{ SAS 3} & 0 & 372 & 75,15 & 88 & 73,95 & 100,00 \\
\hline & 1 & 123 & 24,85 & 31 & 26,05 & 100,00 \\
\hline & Total & 495 & 100,00 & 119 & 100,00 & 100,00 \\
\hline \multirow{3}{*}{ SAS 4} & 0 & 393 & 79,39 & 95 & 79,83 & 100,00 \\
\hline & 1 & 102 & 20,61 & 24 & 20,17 & 100,00 \\
\hline & Total & 495 & 100,00 & 119 & 100,00 & 100,00 \\
\hline \multirow{3}{*}{ COMITE } & 0 & 200 & 40,40 & 60 & 50,42 & 89,67 \\
\hline & 1 & 295 & 59,60 & 69 & 57,98 & 94,49 \\
\hline & Total & 495 & 100,00 & 129 & 108,40 & 92,25 \\
\hline \multirow{3}{*}{ GRI } & 0 & 84 & 16,97 & 30 & 25,21 & 81,83 \\
\hline & 1 & 411 & 83,03 & 101 & 84,87 & 93,51 \\
\hline & Total & 495 & 100,00 & 131 & 110,08 & 90,84 \\
\hline \multirow{3}{*}{ SET } & 0 & 322 & 65,05 & 77 & 64,71 & 100,00 \\
\hline & 1 & 173 & 34,95 & 42 & 35,29 & 100,00 \\
\hline & Total & 495 & 100,00 & 119 & 100,00 & 100,00 \\
\hline
\end{tabular}

Tabela 2 - Variação between e within das variáveis binárias (H1, H3 e H5)

Fonte: Dados da pesquisa.

Conforme a categoria geral, o total de RS assegurados no período pesquisado foi de 199; já, pela categoria between, pelo menos 50 empresas asseguraram o relatório em algum dos anos analisados no estudo. Como a amostra é formada por 119 empresas, percebe-se que na categoria 
between algumas delas mudaram de posição durante o período de 2012 a 2016, por exemplo, a Elektro Redes S.A assegurou seu relatório somente em 2013 e a Even Construtora e Incorporadora S.A deixou de assegurar o RS em 2016. No que se refere ao Setor Ambientalmente Sensível (SAS), destaca-se que nenhuma das empresas foi classificada de acordo com pequeno potencial (SAS 2) de poluição e grau de utilização de recursos naturais (PPGU). A Tabela 3 apresenta a quantidade de empresas que asseguraram os seus relatórios durante o período de análise.

Tabela 2 - Quantidade de empresas que asseguram os RS

\begin{tabular}{ccccccccccc}
\hline \multicolumn{1}{c}{ Opção/Ano } & $\mathbf{2 0 1 2}$ & $\mathbf{\%}$ & $\mathbf{2 0 1 3}$ & $\mathbf{\%}$ & $\mathbf{2 0 1 4}$ & $\mathbf{\%}$ & $\mathbf{2 0 1 5}$ & $\mathbf{\%}$ & $\mathbf{2 0 1 6}$ & $\mathbf{\%}$ \\
\hline Asseguram & 37 & 38,14 & 38 & 37,25 & 42 & 42,42 & 44 & 42,72 & 38 & 40,43 \\
Não asseguram & 60 & 61,86 & 64 & 62,75 & 57 & 57,58 & 59 & 57,28 & 56 & 59,57 \\
\hline \multicolumn{1}{c}{ Total } & 97 & 100 & 102 & 100 & 99 & 100 & 103 & 100 & 94 & 100 \\
\hline
\end{tabular}

Fonte: Dados da pesquisa.

Conforme ilustra a Tabela 3, mais da metade das empresas não asseguraram os RS, apontando para uma leve expressão de crescimento desta prática durante os anos analisados. Em média 40,20\% das empresas asseguraram os RS, enquanto 59,80\% delas não a fizeram. Ademais, a escolha da prestadora da garantia pelas empresas que asseguraram seus RS é demostrada na Tabela 4.

Tabela 3 - Prestadoras da garantia

\begin{tabular}{cccccccc}
\hline Prestadora da Garantia & $\mathbf{2 0 1 2}$ & $\mathbf{2 0 1 3}$ & $\mathbf{2 0 1 4}$ & $\mathbf{2 0 1 5}$ & $\mathbf{2 0 1 6}$ & Total de Assegurações \\
\hline Auditoria & 26 & 28 & 34 & 36 & 31 & 155 \\
Certificadora & 6 & 5 & 7 & 6 & 6 & 30 \\
Especialista & 4 & 4 & 1 & 2 & 1 & 12 \\
Certificadora e Auditoria & 0 & 1 & 0 & 0 & 0 & 1 & 1 \\
Especialista e Auditoria & 1 & 0 & 0 & 0 & 0 & $\mathbf{1 9 9}$ \\
\hline Total & $\mathbf{3 7}$ & $\mathbf{3 8}$ & $\mathbf{4 2}$ & $\mathbf{4 4}$ & $\mathbf{3 8}$ & \\
\hline
\end{tabular}

Fonte: Dados da pesquisa.

A Tabela 4 demonstra que as empresas tiveram seus RS assegurados tanto por empresas de auditoria, como por empresas certificadoras e especialistas. Observa-se que a escolha por empresas de auditoria é prevalente dentre as demais prestadoras de garantia em todos os anos da pesquisa, sendo responsáveis por $155(77,89 \%)$ das 199 assegurações realizadas. Destaca-se que a instituição financeira Bradesco S.A, além de adotar os procedimentos realizados pelas empresas de auditoria contábil, considerou ao mesmo tempo as opiniões e recomendações de firmas mais especializadas em sustentabilidade.

A análise de decomposição de variância between e within para as variáveis contínuas, referentes às hipóteses $\mathrm{H} 2$ e H5, e de controle é exibida na Tabela 5, sendo a variável Rentabilidade (RENT) a que possui menor dispersão em relação às demais variáveis, enquanto a variável Tamanho (TAM) apresenta maior amplitude entre as observações.

Com relação ao percentual de empresas que pertencem ao mesmo setor que asseguram o RS (PRESET), destaca-se que o setor Tecnologia é o único que não endossa essa prática. Já os setores de Saúde, Financeiros e Outros, Materiais Básicos, Telecomunicações e Consumo não Cíclico obtiveram o percentual em mais de $50 \%$ em todos os anos analisados. Já Consumo Cíclico, Bens Industriais, Petróleo, Gás e Biocombustíveis e Utilidade Pública obtiveram percentuais que variaram entre $8 \%$ e $43 \%$.

Ainda, ao considerar a variância dos desvios-padrões da média dos regressores, nota-se que a variação entre empresas é maior do que a variação para cada empresa ao longo do tempo. Isso indica que as alterações em $\mathrm{X}$ ao longo do tempo são menores que do que o comportamento de $\mathrm{X}$ entre os indivíduos para cada cross-section (Fávero et al., 2009). Essa evidência reforça o argumento de que a análise em painel é potencialmente mais informativa, já que esse comportamento possui maior variabilidade ao longo do tempo. 
Tabela 5 - Decomposição de variância between e within para as variáveis contínuas

\begin{tabular}{ccccccc}
\hline Variáveis & Decomposição & Média & Desvio Padrão & Mínimo & Máximo & Observações \\
\hline \multirow{2}{*}{ PRESET } & Geral & & 0,1649 & 0 & 1 & $\mathrm{~N}=495$ \\
& Between & 0,4023 & 0,1697 & 0 & 1 & $\mathrm{n}=119$ \\
& Within & & 0,0523 & 0,2487 & 0,6413 & $\mathrm{~T}-\mathrm{bar}=4,1596$ \\
& Geral & & 0,3791 & -0.7768 & 5,0516 & $\mathrm{~N}=495$ \\
CRESC & Between & 0,1160 & 0,1869 & -0.3668 & 0,9601 & $\mathrm{~N}=119$ \\
& Within & & 0,3333 & $-1,4684$ & 4,2351 & $\mathrm{~T}-\mathrm{bar}=4,1596$ \\
& Geral & & 1,6434 & 19,6627 & 27,9939 & $\mathrm{~N}=495$ \\
TAM & Between & 23,1359 & 1,6262 & 19,7010 & 27,9196 & $\mathrm{n}=119$ \\
& Within & & 0,2023 & 22,2619 & 24,7134 & $\mathrm{~T}-\mathrm{bar}=4,1596$ \\
& geral & & 0,0768 & $-0,5373$ & 0,3284 & $\mathrm{~N}=495$ \\
RENT & between & 0,0319 & 0,0665 & $-0,2767$ & 0,2155 & $\mathrm{n}=119$ \\
& within & & 0,0446 & $-0,2558$ & 0,2915 & $\mathrm{~T}-\mathrm{bar}=4,1596$ \\
& geral & & 0,2128 & $9,94 \mathrm{e}-10$ & 1.4168 & $\mathrm{~N}=495$ \\
ENDIV & between & 0,6313 & 0,2114 & $9,94 \mathrm{e}-10$ & 1.2019 & $\mathrm{n}=119$ \\
& within & & 0,0645 & 0,3291 & 1.2512 & $\mathrm{~T}-\mathrm{bar}=4,1596$ \\
\hline
\end{tabular}

Fonte: Dados da pesquisa.

Por meio da matriz de correlação, exibida na Tabela 6 , verifica-se que a maior correlação encontrada entre as variáveis de controle é de -0,42 entre Endividamento e Rentabilidade, representando uma correlação moderada $(0,40$ a 0,69$)$. As correlações restantes são consideradas baixas $(0,01$ a 0,39$)$, e isso indica que o modelo não apresenta problemas de multicolinearidade.

Tabela 6 - Matriz de correlação entre as variáveis

\begin{tabular}{|c|c|c|c|c|c|c|c|c|c|c|c|}
\hline & \begin{tabular}{l}
0 \\
I \\
$\approx$ \\
\multirow{2}{*}{}
\end{tabular} & $\sum_{\sim}^{\infty}$ & $\begin{array}{l}\nabla \\
\stackrel{4}{*}\end{array}$ & 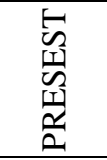 & $\sum_{0}^{\stackrel{U}{\mid}}$ & 胥 & 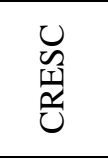 & $\sum_{\in}$ & 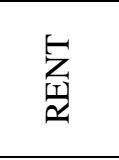 & $\sum_{\text {至 }}$ & 窗 \\
\hline ASSEG & 1 & & & & & & & & & & \\
\hline SAS 3 & $-0.09^{*}$ & 1 & & & & & & & & & \\
\hline SAS 4 & 0.03 & $-0.29^{* *}$ & 1 & & & & & & & & \\
\hline PRESET & $0.34^{* *}$ & $-0.16^{\text {** }}$ & $0.17^{* *}$ & 1 & & & & & & & \\
\hline COMITE & $0.24^{* *}$ & 0.03 & 0.03 & 0.04 & 1 & & & & & & \\
\hline GRI & $0.34^{* *}$ & -0.06 & -0.02 & $0.17^{* *}$ & $0.18^{* *}$ & 1 & & & & & \\
\hline CRESC & -0.05 & 0.01 & -0.05 & 0.01 & 0.06 & 0.02 & 1 & & & & \\
\hline TAM & $0.38^{* *}$ & -0.01 & -0.05 & $0.32^{* *}$ & $0.18^{* *}$ & $0.20^{* *}$ & 0.02 & 1 & & & \\
\hline RENT & 0.01 & $0.10^{*}$ & $-0.15^{* *}$ & -0.07 & -0.08 & -0.03 & $0.20^{* *}$ & -0.06 & 1 & & \\
\hline ENDIV & 0.07 & $-0.12^{\text {** }}$ & -0.04 & 0.06 & 0.04 & 0.05 & 0.01 & $0.25^{* *}$ & $-0.42^{* *}$ & 1 & \\
\hline SET & $0.13^{\text {** }}$ & -0.02 & $-0.32^{* *}$ & $0.19^{* *}$ & $0.17^{* *}$ & 0.04 & $0.10^{*}$ & $0.24^{* *}$ & 0.03 & $0.15^{\text {** }}$ & 1 \\
\hline
\end{tabular}

Nota: * e ** correspondem a 5\% e 1\% de nível de significância, respectivamente.

Fonte: Dados da pesquisa.

\subsection{Modelo de regressão logística em painel}

O emprego da técnica de regressão logística em painel na forma de modelo de efeitos mistos para testar as hipóteses estabelecidas nesta pesquisa busca alcançar o objetivo geral de identificar os fatores que podem influenciar a ocorrência da asseguração dos RS no Brasil, bem como verificar a relação existente entre eles. A Tabela 7 fornece os resultados da regressão logística em painel.

A análise dos coeficientes indica efeito significativo e positivo para as variáveis Pressão do Setor (PRESET); Comitê de Sustentabilidade (COMITE); GRI; e Tamanho (TAM) aos níveis de 5\% e $1 \%$. Desse modo, dentre as dez variáveis incluídas neste modelo, quatro delas podem ser consideradas para explicar a probabilidade de uma empresa assegurar o RS. Estes resultados confirmam as hipóteses $\mathrm{H} 2(17,23, \mathrm{p}<0,01) \mathrm{H} 3(5,05, \mathrm{p}<0,01)$ e $\mathrm{H} 4(9,81, \mathrm{p}<0,05)$ de que a 
probabilidade de adoção da asseguração dos RS é afetada positivamente pela Pressão do Setor, existência do Comitê de Sustentabilidade e adesão das diretrizes da GRI. Também confirmou que empresas maiores (TAM, 2,61, p<0,01) tendem a ter os recursos financeiros necessários para custear os serviços de asseguração externa.

Tabela 7 - Resultados da regressão logística em painel

\begin{tabular}{|c|c|c|c|c|c|}
\hline \multirow{5}{*}{ ASSEG } & \multicolumn{5}{|c|}{ Random effects } \\
\hline & Grupos & Nome & Variance & \multicolumn{2}{|c|}{ Erro Padrão } \\
\hline & Empresas & (Intercept) & 68.64 & \multicolumn{2}{|c|}{8.285} \\
\hline & \multicolumn{5}{|c|}{ Fixed effects } \\
\hline & Odds Ratio & Coeficiente & Erro Padrão & p-value & Significância \\
\hline (Intercept) & & $-77,2733$ & 24,7678 & 0,0018 & $* *$ \\
\hline SAS 3 & 0,011574 & $-4,4590$ & 2,6737 & 0,0954 & \\
\hline SAS 4 & 0,974194 & $-0,0261$ & 2,9779 & 0,9930 & \\
\hline PRESET & 30439340 & 17,2312 & 5,7060 & 0,0025 & $* *$ \\
\hline COMITE & 156.5556 & 5,0534 & 1,7995 & 0,0049 & $* *$ \\
\hline GRI & 18326 & 9,8161 & 5,0064 & 0,0499 & $*$ \\
\hline CRESC & 0,4157 & $-0,8778$ & 1,1113 & 0,4296 & \\
\hline TAM & 13,7005 & 2,6174 & 0,9733 & 0,0071 & $* *$ \\
\hline RENT & 0,3354 & $-1,0923$ & 5,4912 & 0,8423 & \\
\hline ENDIV & 0,0019 & $-6,2917$ & 3,4675 & 0,0696 & \\
\hline SET & 0,7340 & $-0,3093$ & 2,4429 & 0,8992 & \\
\hline Observações & & & 495 & & \\
\hline Log likelihood & & & $-120,2$ & & \\
\hline Prob > chi 2 & & & 0,0000 & & \\
\hline
\end{tabular}

Nota: * e ** correspondem a 5\% e 1\% de nível de significância, respectivamente. O modelo logístico classificou $66,87 \%$ das observações analisadas $([180+151] / 495=66,87)$. A área sob a curva ROC $=0,8292$. Teste de Hosmer-Lemeshow $=1843,03$ ( $p$-value de 0,5283) indicou a existência de associação e considera que o modelo apresenta um bom ajuste.

Fonte: Dados da pesquisa.

As relações entre asseguração dos RS e Setor Ambientalmente Sensível (SAS), Oportunidade de Crescimento (CRESC), Rentabilidade (RET), Endividamento (ENDIV) e Setor (SET - bancos e energia elétrica) não apresentaram significância estatística ( $\mathrm{p}<0,01$ e 0,05$)$ nem coeficiente positivo, como pressuposto pelas H1, H5 e pelos estudos apontados na literatura (Simnett et al., 2009; Kolk \& Perego, 2010; Ruhnke \& Gabriel, 2013; Cho et al., 2014; Branco et al., 2014; Peters \& Romi, 2014; Martínez-Ferrero \& García-Sánchez, 2016, 2017). Assim, não há evidências suficientes para afirmar que a asseguração dos RS é afetada positivamente pelo impacto ambiental causado pelo setor de atividade e pelo constante crescimento das empresas.

A razão de chance (odds ratio) diz a respeito à mudança na chance de ocorrência do evento ao se alterar em uma unidade esta mesma variável, preservadas as demais condições constantes (Fávero et al., 2009). Nesta lógica, a cada aumento de uma unidade no tamanho da empresa, multiplica-se em 13,7 vezes a chance do RS ser assegurado externamente.

Quanto à PRESET, no caso das empresas confrontadas com maior Pressão do Setor, aumentase em 30.439.340 vezes a chance de submissão do RS à verificação externa. Para COMITE, quando a empresa dispõe de um comitê que trata dos assuntos relativos à sustentabilidade, multiplica-se em 156,5 vezes a probabilidade de ocorrência da asseguração do RS. Enquanto GRI, as empresas que elaboram seus RS de acordo com as diretrizes GRI têm a chance de assegurá-los aumentada em 18.326 vezes.

\subsection{Discussão dos Resultados}

O estudo identificou que, dentre as 119 empresas componentes da amostra, em média 40,20\% das empresas asseguraram os RS. Nota-se que, como apontado no estudo de Silveira et al. (2019), 
por mais que a divulgação dos RS tenha se estabelecido como uma prática comum entre as empresas, a asseguração destes ainda não é adotada pela maioria das empresas analisadas neste estudo, já que o processo de asseguração é uma prática voluntária. Enfatiza-se que esse comportamento possa estar relacionado à percepção da empresa sobre os custos e benefícios da asseguração externa, pois as empresas somente tomam por relevante tal prática, quando os benefícios derivados dela são percebidos, compensando os custos associados (Simnett et al., 2009).

Tem-se, ainda, um predomínio das empresas de auditoria independente (empresas de contabilidade) em relação às demais prestadoras de garantia para todos os anos da pesquisa, sendo responsáveis por $77,89 \%$ dos trabalhos realizados. Estes resultados se assemelham aos encontrados por Silveira et al. (2019) de que as firmas de auditoria contábil independente possuem certa credibilidade no ramo de verificação e validação de informações em contexto brasileiro. Esses resultados são contrários aos achados de Simnett et al., (2009) de que as empresas não fazem distinção pela natureza do provedor.

Quanto aos resultados obtidos por meio da regressão logística em painel, a hipótese H1 que previa a relação positiva entre a probabilidade de adoção da asseguração dos RS e o impacto ambiental causado pelo setor de atividade não foi confirmada devido a não significância no modelo e coeficientes negativos das variáveis SAS 3 e SAS 4. Tal resultado segue em sentido oposto das afirmações e dos achados de Simnett et al. (2009), Peters e Romi (2014) e Cho et al. (2014) de que, diante do risco ambiental, as empresas, podem mostrar disposição em assegurar o RS na tentativa de reforçar sua legitimidade perante seus stakeholders. Contudo, justificativas para esse resultado podem estar relacionadas à especificidade da amostra e a proxy utilizada. Dos relatórios assegurados, mais de 54\% (270) pertenciam a empresas que não foram classificadas nos níveis de impacto ambiental, SAS 1, estabelecidos na Lei $n^{\circ} 10.165 / 2000$ (Brasil, 2000). Como a referida lei tem quase 20 anos, a mensuração de Setor Ambientalmente Sensível por tal pode não refletir a diversidade de setores de atuação das empresas atualmente.

A hipótese $\mathrm{H} 2$ buscou confirmar se há relação positiva entre a probabilidade de adoção da asseguração dos RS e a pressão exercida pelo setor de atividade, a qual foi confirmada ao nível de significância de $1 \%$. Os resultados corroboram as pesquisas de Peters e Romi (2014) e de MartínezFerrero e García-Sánchez (2016) de que a legitimidade das empresas resulta de práticas amplamente adotada em seu setor, demonstrando um comportamento derivado de forças miméticas.

Quanto à hipótese $\mathrm{H} 3$, que previa a relação positiva entre a probabilidade de adoção da asseguração dos RS e a existência do Comitê de Sustentabilidade, também se confirmou ao nível de significância de 1\%. Os resultados são semelhantes aos de Ruhnke e Gabriel (2013) e MartínezFerrero e García-Sánchez (2017), pois confirmam que a atividade do comitê influencia na probabilidade de ocorrência da asseguração. Dessa forma, os comitês de sustentabilidade cumprem um papel importante na tentativa das empresas em buscar reforço da legitimidade alcançada pela divulgação de suas informações socioambientais, enfatizando atribuir credibilidade a essas informações por meio da asseguração externa.

A hipótese $\mathrm{H} 4$ que predizia o efeito positivo da adesão das Diretrizes da GRI para elaboração dos RS na probabilidade de adoção da asseguração foi aceita $(\mathrm{p}<0,05)$. As evidências geradas neste estudo confirmam a associação verificada por Silveira et al. (2019) entre as diretrizes GRI e asseguração dos RS. Ruhnke e Gabriel (2013) também atestam estes resultados ao identificarem como constructo de sinalização os níveis de aplicação da GRI como fator influenciador na garantia externa voluntária das empresas da Alemanha, Holanda e Grã-Bretanha. Assim, a emissão de relatórios com base na GRI afeta a credibilidade dos relatórios positivamente (Lock \& Seele, 2016), o que contribui para uma maior percepção sobre o nível de legitimidade alcançada pelas empresas.

A hipótese $\mathrm{H} 5$ objetivou testar a relação entre a probabilidade de adoção da asseguração e a Oportunidade de Crescimento das empresas. O resultado para a variável CRESC não foi significante, o que corrobora com os achados de Kuzey e Uyar (2017) ao não confirmarem que as empresas em 
constante crescimento tendem a assegurar os RS. Ao que parece os RS não são divulgados e assegurados pelas empresas como forma ou mecanismo de legitimar a continuidade de seus negócios.

Com respeito a variáveis de controle Tamanho (TAM), Rentabilidade (RENT), Endividamento (ENDIV) e Setor (SET), somente se confirmou a significância e o sinal esperado para TAM. Portanto, o tamanho ou porte da empresa se configura em uma importante característica da empresa que pode condicionar a ocorrência da asseguração dos RS, conforme os estudos de estudos de Simnett et al., (2009), Branco et al. (2014), Peters e Romi (2014), Martínez-Ferrero e GarcíaSánchez (2016), Luna et al. (2017), Martínez-Ferrero e García-Sánchez (2017). Ou seja, a asseguração dos RS apresenta uma maior chance de ocorrência em empresas maiores e, consequentemente, mais evidentes no mercado.

\section{CONSIDERAÇÕES FINAIS}

Diante da proposição de que a asseguração externa tem sido empregada no sentido de atribuir credibilidade aos Relatórios de Sustentabilidade (RS), de modo a influenciar o nível de legitimidade das organizações, este estudo objetivou identificar os fatores que podem influenciar a ocorrência da asseguração dos RS no Brasil, a partir de variáveis consubstanciadas na Teoria da Legitimidade. Para isso, as variáveis explicativas selecionadas com base na literatura pré-existente foram Setor Ambientalmente Sensível (SAS), Pressão do setor (PRESET), Comitê de Sustentabilidade (COMITE), Global Reporting Initiative (GRI) e Oportunidade de Crescimento (CRESC). Além das variáveis corporativas de controle Tamanho, Rentabilidade, Endividamento e Setor. Incluíram-se na análise todas as companhias listadas na B3 em 2017 que divulgaram RS entre os anos de 2012 a 2016.

O modelo especificado para esta pesquisa consistiu em modelo logístico em painel de efeitos mistos. Dessa forma, a presente pesquisa identificou que Pressão do Setor, Comitê de Sustentabilidade, as Diretrizes da GRI e Tamanho são variáveis que podem influenciar a probabilidade ocorrer asseguração externa dos RS. A busca das empresas por legitimidade por meio dessa prática ocorre, portanto, em empresas maiores e mais evidentes e pode resultar de práticas amplamente adotada em seu setor, do monitoramento das questões de sustentabilidade exercido por comitês especializados ligados à estrutura de governança corporativa e da adoção de práticas de relato que atribuem qualidade e credibilidade aos RS.

Com base nos resultados, acredita-se que, em razão do objetivo da divulgação dos RS consistir em alcançar ou proteger a legitimidade organizacional, a asseguração externa dos RS contribui para aumentar o nível dessa legitimidade à medida que esta prática se desenvolve em ambientes onde as empresas demonstram melhor as suas práticas de sustentabilidade; afinal, o principal produto do processo contábil consiste na informação, a qual, em um contexto onde diversos usuários se utilizam da mesma informação, é percebida como bem comum que necessita ser relevante e credível a suas partes interessadas.

Em suma, esta pesquisa contribui para a discussão sobre a temática de asseguração dos RS, sobretudo as motivações que levam as empresas a adotar essa prática devido à sua natureza voluntária, por exemplo: o que influencia? Em que condições ocorre? Como as empresas a realizam? Dessa maneira, este trabalho visou a retratar a realidade das empresas brasileiras com a suposição de que a principal motivação envolve fatores ligados à legitimidade de suas operações, e, consequentemente, à construção de sua reputação corporativa perante a sociedade e os demais stakeholders. Outrossim, este estudo demonstra diferentes resultados para setores que enfrentam maior risco social e ambiental e asseguração dos RS apontados pela literatura, o que abre lacunas para futuras pesquisas levantarem novas evidências para discussão dessa característica. Também explora novas proxys diferentes daquelas estudadas em diferentes contextos e variáveis que carecem de mais estudo como a Oportunidade de Crescimento. Ainda, contribui para discutir o papel da Contabilidade na articulação entre os impactos socioambientais das atividades empresariais e o desempenho econômico por meio dos relatórios de sustentabilidade, em conformidade com sua função de mensuração, reconhecimento, 
controle divulgação e verificação externa; e esta última pode ser atribuída a empresas de auditoria independente, como evidencia este e os demais estudos.

Este trabalho teve como principais limitações à forma voluntária e diversificada de como as empresas relatam suas informações socioambientais, bem como a falta de padrão quanto à periocidade das divulgações dos RS. Por isso, a pesquisa trabalhou somente com relatórios disponíveis durante o período de coleta. Quanto à amostra e ao período, ressalta-se que os resultados aqui gerados se aplicam somente às empresas estudadas, e não devem ser generalizados para outros períodos ou organizações. Ademais, os fatores identificados neste estudo não esgotam todas as variáveis intervenientes que possam influenciar adoção da prática de assegurar os RS.

Desse modo, sugere-se, para futuras pesquisas, aumentar o período de análise, o que proporcionaria o aumento das observações, e assim melhores estimações nas análises estatísticas. Recomenda-se, também, identificar novos fatores ou até avaliar os mesmos empregados neste estudo utilizando proxys distintas das que foram empregadas nesta pesquisa - por exemplo, o fator relacionado ao SAS pode ser testado de outra forma, conforme aponta a literatura, bem como estabelecer uma análise de abordagem internacional e comparativa com empresas de outros países.

Propõe-se, ainda, diante da baixa incidência de asseguração dos RS da amostra, o estudo mais aprofundado da percepção dos stakeholders, das empresas e dos provedores de garantia sobre a asseguração dos RS de modo a avaliar os custos e benefícios propostos por ela. É necessário estudar as reais motivações por detrás desta prática, já que as empresas podem envolver-se em práticas de responsabilidade social para cobrir o impacto de uma má conduta corporativa.

Nesse sentido, vale ressaltar a discricionariedade que as empresas possuem em relação ao escopo da garantia, bem como a falta de normas, orientações e regulamentação na área. Além do mais, o conteúdo da garantia pode variar de acordo com as preferências do assegurado e do assegurador, o que pode levar a não compreensão por grande parte dos usuários da informação. E essas condições levantam questionamentos sobre a qualidade dos trabalhos de asseguração externa.

\section{REFERÊNCIAS}

Adams, CA, Hill, WY e Roberts, CB (1998). Práticas de relatórios sociais corporativos na Europa Ocidental: legitimando o comportamento corporativo?. The British Accounting Review , 30 (1), 1-21.

Agência Nacional de Energia Elétrica (2016). Despacho no 3.034/2006. 2006. Disponível em: http://www. aneel.gov.br. Acesso em: 19 maio.

Artiach, T., Lee, D., Nelson, D., \& Walker, J. (2010). The determinants of corporate sustainability performance. Accounting \& Finance, 50(1), 31-51.

Arvidsson, S. (2010). Communication of corporate social responsibility: A study of the views of management teams in large companies. Journal of Business Ethics, 96(3), 339-354.

Banco Central (2014). Resolução CMN $n^{o}$ 4.327/2014. Implementação da Política de Responsabilidade Socioambiental pelas instituições financeiras e demais instituições autorizadas a funcionar pelo Banco Central do Brasil. Brasília: BCB.

Branco, M. C., Delgado, C., Gomes, S. F., \& Eugénio, T. C. P. (2014). Factors influencing the assurance of sustainability reports in the context of the economic crisis in Portugal. Managerial Auditing Journal.

Brasil (2019). Lei $n^{o} 10.165$, de 27 de dezembro de 2000. Altera a Lei $n^{\circ} 6.938$, de 31 de agosto de 1981, que dispõe sobre a Política Nacional do Meio Ambiente, seus fins e mecanismos de formulação e aplicação, e dá outras providências. Disponível em: http://www.planalto.gov.br/ccivil_03/Leis/L10165.htm. Acesso em: 27 ago.

Brasil, Balcão e Bolsa (B3) (2011). Comunicado Externo 017/2011-DP. Proposta de Relate ou Explique sobre os relatórios de sustentabilidade para as empresas brasileiras. 23 dez. 2011. Disponível em: http://www.b3.com.br/data/files/83/51/A5/A2/02121510FE0C840592D828A8/CE-017-2011Proposta-de-adocao-ao-modelo-Relate-ou-Explique.pdf. Acesso em: 27 ago de 2017.

Cho, C. H., Michelon, G., Patten, D. M., \& Roberts, R. W. (2014). CSR report assurance in the USA: an empirical investigation of determinants and effects. Sustainability Accounting, Management and Policy Journal. 
Deegan, C. (2002). The legitimising effect of social and environmental disclosures-a theoretical foundation. Accounting, Auditing \& Accountability Journal, 15(3), 282-311.

Deephouse, D. L., \& Carter, S. M. (2005). An examination of differences between organizational legitimacy and organizational reputation. Journal of management Studies, 42(2), 329-360.

Economática®. [s. d.]. Disponível em: https://economatica.com. Acesso em: 22 set. 2017.

Eugenio, T. P. (2010). Avanços na divulgação de informação social e ambiental pelas empresas e a teoria da legitimidade. Revista Universo Contábil, 6(1), 102-118.

Fávero, L. P. L., Belfiore, P. P., Silva, F. L. D., \& Chan, B. L. (2009). Análise de dados: modelagem multivariada para tomada de decisões.

Global Initiative Reporting (GRI) (2017). Focal Point Brazil. Disponível em: https://www.globalreporting.org/network/regional-networks/gri-focal-points/focal-pointbrazil/Pages/default.aspx. Acesso em: 20 set. 2017.

Gürtürk, A., \& Hahn, R. (2016). An empirical assessment of assurance statements in sustainability reports: smoke screens or enlightening information?. Journal of Cleaner Production, 136, 30-41.

Gurvitsh, N., \& Sidorova, I. (2012). Survey of sustainability reporting integrated into annual reports of Estonian companies for the years 2007-2010: based on companies listed on Tallinn Stock Exchange as of October 2011. Procedia Economics and Finance, 2, 26-34.

Hemingway, C. A., \& Maclagan, P. W. (2004). Managers' personal values as drivers of corporate social responsibility. Journal of business ethics, 50(1), 33-44.

Hodge, K., Subramaniam, N., \& Stewart, J. (2009). Assurance of sustainability reports: Impact on report users' confidence and perceptions of information credibility. Australian accounting review, 19(3), 178-194.

Hox, J. J., Moerbeek, M., \& Van de Schoot, R. (2010). Multilevel analysis: Techniques and applications. Routledge.

Hummel, K., Schlick, C., \& Fifka, M. (2019). The role of sustainability performance and accounting assurors in sustainability assurance engagements. Journal of Business Ethics, 154(3), 733-757.

Islam, M. A., \& Deegan, C. (2008). Motivations for an organisation within a developing country to report social responsibility information. Accounting, Auditing \& Accountability Journal.

Kim, Y., Park, M. S., \& Wier, B. (2012). Is earnings quality associated with corporate social responsibility?. The accounting review, 87(3), 761-796.

Kolk, A., \& Perego, P. (2010). Determinants of the adoption of sustainability assurance statements: An international investigation. Business strategy and the environment, 19(3), 182-198.

KPMG. International survey of corporate responsibility reporting. Amsterdam, 2011. Disponível em: http://www.kpmg.com/Global/en/IssuesAndInsights/ArticlesPublications/ corporateresponsibility/Documents /2011-survey.pdf. Acesso em: 20 nov. 2016.

Kuzey, C., \& Uyar, A. (2017). Determinants of sustainability reporting and its impact on firm value: Evidence from the emerging market of Turkey. Journal of cleaner production, 143, 27-39.

Lamberton, G. (2005, March). Sustainability accounting - a brief history and conceptual framework. In Accounting forum (Vol. 29, No. 1, pp. 7-26). Taylor \& Francis.

Lock, I., \& Seele, P. (2016). The credibility of CSR (corporate social responsibility) reports in Europe. Evidence from a quantitative content analysis in 11 countries. Journal of Cleaner Production, 122, 186200.

Lozano, R., \& Huisingh, D. (2011). Inter-linking issues and dimensions in sustainability reporting. Journal of cleaner production, 19(2-3), 99-107.

Luna, T. A. U; Gomes, S. M. da S.; Martinez, A. L.; Cordeiro Filho, J. B.; Bomfim, R. F. F. (2017). Auditoria dos Relatórios de Sustentabilidade: um estudo com Empresas Listadas na B3. In: Encontro Internacional sobre Gestão Empresarial e Meio Ambiente (ENGEMA), 19. São Paulo. Anais eletrônicos [...]. São Paulo: USP, 2017. Disponível em: http://engemausp.submissao.com.br/19/anais/arquivos/43.pdf. Acesso em: 25 ago. 2017.

Martínez-Ferrero, J., \& García-Sánchez, I. M. (2017). Coercive, normative and mimetic isomorphism as determinants of the voluntary assurance of sustainability reports. International Business Review, 26(1), $102-118$.

Meyer, J. W., \& Rowan, B. (1977). Institutionalized organizations: Formal structure as myth and ceremony. American journal of sociology, 83(2), 340-363.

Moroney, R., Windsor, C., \& Aw, Y. T. (2012). Evidence of assurance enhancing the quality of voluntary environmental disclosures: An empirical analysis. Accounting \& Finance, 52(3), 903-939. 
O'Dwyer, B., Owen, D., \& Unerman, J. (2011). Seeking legitimacy for new assurance forms: The case of assurance on sustainability reporting. Accounting, Organizations and Society, 36(1), 31-52.

Parsons, Talcott (1960). Structure and process in modern societies. Glencoe, Illinois: The Free Press.

Patten, D. M. (2002). The relation between environmental performance and environmental disclosure: a research note. Accounting, organizations and Society, 27(8), 763-773.

Perego, P., \& Kolk, A. (2012). Multinationals' accountability on sustainability: The evolution of third-party assurance of sustainability reports. Journal of Business Ethics, 110(2), 173-190.

Peters, G. F., \& Romi, A. M. (2015). The association between sustainability governance characteristics and the assurance of corporate sustainability reports. Auditing: A Journal of Practice \& Theory, 34(1), 163198.

Ruhnke, K., \& Gabriel, A. (2013). Determinants of voluntary assurance on sustainability reports: an empirical analysis. Journal of Business Economics, 83(9), 1063-1091.

Sancovschi, M., \& Silva, A. H. C. (2010). Evidenciação social corporativa: estudo de caso da empresa Petróleo Brasileiro SA. Sociedade, Contabilidade e Gestão, 1(1).

Silveira, G. B., Alberton, L., \& Vicente, E. F. R. (2017). O Estado da Arte da Asseguração dos Relatórios de Sustentabilidade: Um Estudo das Empresas do Índice de Sustentabilidade Empresarial (ISE) 2016. Environmental \& Social Management Journal/Revista de Gestão Social e Ambiental, 11(2).

Silveira, G. B., da Silva Ferreira, J., Farias, R. B., Van Bellen, H. M., \& Rover, S. (2019). Assegurar ou não assegurar? Eis a questão: uma análise dos fatores associados à asseguração dos relatórios de sustentabilidade. RACE-Revista De Administração, Contabilidade E Economia, 18(2), 259-280.

Simnett, R., Vanstraelen, A., \& Chua, W. F. (2009). Assurance on sustainability reports: An international comparison. The accounting review, 84(3), 937-967.

Suchman, M. C. (1995). Managing legitimacy: Strategic and institutional approaches. Academy of management review, 20(3), 571-610. 